\title{
Vomiting in a toddler - An unsuspected cause
}

\author{
N Rashmi ${ }^{1}$, Devesh Bhaskar Yerrapragada ${ }^{2}$, M G Anil Kumar ${ }^{3}$ \\ From ${ }^{l}$ Associate Professor, ${ }^{2}$ Junior Resident, Department of Pediatrics, ${ }^{3}$ Professor, Department of Pediatric Surgery, JSS Medical College and Hospital, \\ JSSAHER, Mysuru, Karnataka, India
}

\begin{abstract}
Vomiting in young children can be due to a varied etiology ranging from gastritis, urinary tract infection, and metabolic problems to serious life-threatening conditions such as myocarditis, intracranial space occupying lesions, and acute surgical conditions such as intussusception, malrotation, and torsion testis. However, intractable vomiting in a toddler as presented here was due to an unexpected cause as the history was not initially forthcoming for the same. Here, we presented the case of a 21-month-old girl who was admitted with vomiting and low-grade fever, initially suspected to have viral fever/urinary tract infection. All relevant investigations were within normal limits, ruling out metabolic causes. However, on day 3, she showed features of intestinal obstruction for which imaging was done which revealed a mass in the distal ileum, and she was taken up for immediate laparotomy and the ileal mass was excised, which turned out to be a foreign body, that is, three rubber balls. The child recovered completely post-surgery and was discharged three days later. Hence, the lessons learnt from this case would be that, there is no short cut to detailed history taking and meticulous clinical examination; using imaging at the appropriate time helps in timely intervention and life-saving management.
\end{abstract}

Key words: Gastrointestinal foreign body, Ileal mass, Intestinal obstruction, Rubber balls

$\mathrm{F}$ oreign body ingestion most commonly occurs in children aged 6 months to 3 years and accounts for approximately $80 \%$ of all foreign body ingestions in children. A gastrointestinal foreign body can be found in any location of the intestinal tract [1]. To the best of our knowledge, there were three previous reports of an infant having small bowel obstruction due to ingestion of rubber balls [2-4]. Here, we report a case of a 21-months-old child who presented to our hospital with acute onset of vomiting and low-grade fever progressing to subacute intestinal obstruction, eventually to find a foreign body consisting of three rubber balls in the distal ileum.

\section{CASE PRESENTATION}

A 21-month-old developmentally normal female child born out of non-consanguineous marriage presented to our hospital with complaints of vomiting from one day, around 4-5 episodes per day, immediately following feeds. Vomitus contained food particles, was non-projectile, non-bilious, non-foul smelling, and not blood tinged. Child also had low-grade fever of one day duration. There was no history of loose stools, pain abdomen, and abdominal distention or cough, cold, breathlessness, or

\section{Access this article online}

Received - 27 August 2021

Initial Review - 14 September 2021

Accepted - 24 September 2021

DOI: 10.32677/IJCH.2021.v08.i10.3073 burning micturition. Alongside, there was no history of eating contaminated food, or accidental ingestion of a foreign body. Clinical examination revealed normal vital parameters with anthropometric indicators - all below the $5^{\text {th }}$ centile on the growth charts by Centers for Disease Control (CDC), indicating chronic malnutrition. Abdominal examination showed a soft and non-tender abdomen with just palpable liver and a nonpalpable spleen. Other systems were unremarkable. A provisional diagnosis of acute viral gastritis/urinary tract infection was made and child was started on symptomatic treatment after relevant investigations were sent for. These showed $\mathrm{Hb} 11.4 \mathrm{~g}$, total WBC count of $8300 / \mathrm{mm}^{3}$ (Neutrophils: $66 \%$ and lymphocytes: $33 \%$ ), and platelet count 3.12 lakh with peripheral blood smear showing relative neutrophilia. Serum electrolytes, liver function, and renal function tests were within the normal limits. Other metabolic workup including serum ammonia, lactate and arterial blood gas analysis were within the normal limits. Urine routine and microscopic examination was normal. Hence, the child was continued on anti-emetics for 2 days. However, vomiting did not subside and she developed mild abdominal distention on day 3 with sluggish bowel sounds and had not passed stools for 2 days. Serum electrolyte levels and blood glucose were repeated and found normal. Hence, suspecting subacute intestinal obstruction, an ultrasound abdomen was done which

Correspondence to: $\mathrm{N}$ Rashmi, Department of Pediatrics, JSS Medical College Hospital, M.G.Road, Agrahara, Mysuru - 570 004, Karnataka, India. E-mail: dr.rashminagaraj@gmail.com

(C) 2021 Creative Commons Attribution-NonCommercial 4.0 International License (CC BY-NC-ND 4.0). 
showed a well encapsulated round anechoic lesion around $2.5 \times 2.5 \mathrm{~cm}$ in the left hypochondriac region with adjacent bowel showing peristalsis with minimal effusion, with mesenteric lymphadenitis - suggestive of a duplication cyst/artifact. X-ray erect abdomen done showed dilated bowel loops. Pediatric surgery opinion was taken and child was managed conservatively for subacute intestinal obstruction. However, child became lethargic the next day, with increasing abdominal distension. She was kept nil by mouth and was started on intravenous fluids, but abdominal distension continued to increase with sluggish bowel sounds. CT abdomen done on the same day confirmed the mass in the distal ileum with proximal ileal and jejunal dilation. Furthermore, the child continued to have vomiting with two episodes of bilious and feculent vomiting and hence was taken up for laparotomy. During laparotomy, a blackish mass was seen in the distal ileum which was mobile within the lumen around $15-20 \mathrm{~mm}$ from the ileocecal junction (Fig. 1).

Vertical enterotomy was done and objects were dissected. One blackish $4 \times 4 \mathrm{~cm}$ smooth firm rubber ball and 2 translucent multi-colored hemi spherical rubber objects were present which were removed (Fig. 2).

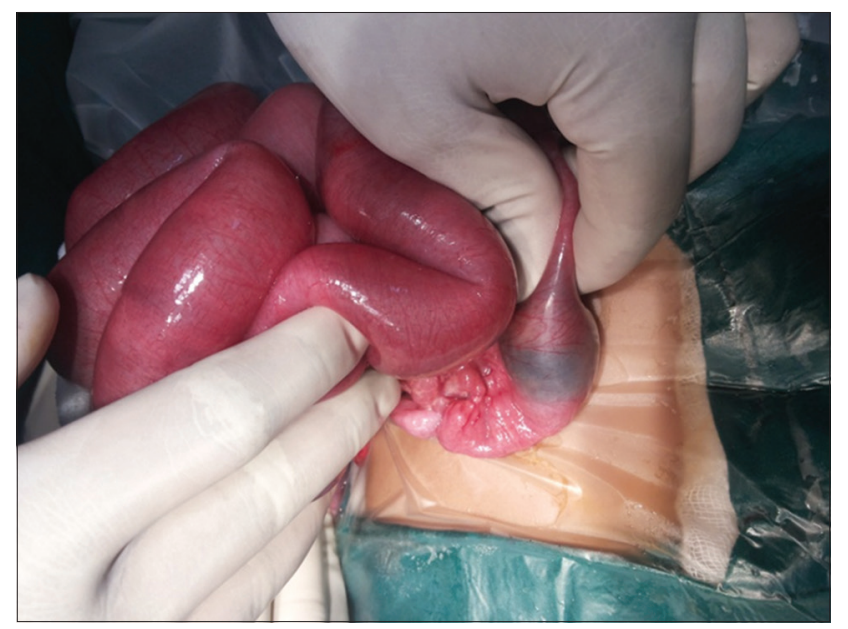

Figure 1: Intra-operative picture showing a blackish mass at the distal ileum

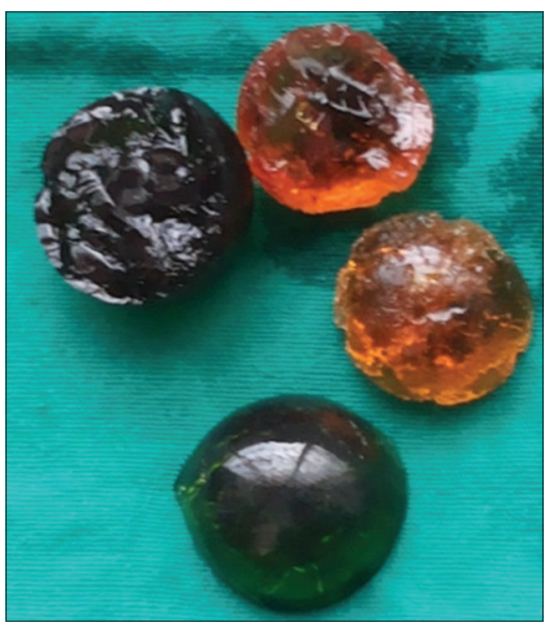

Figure 2: Picture showing the two transparent round objects and one blackish rubber ball removed from the distal ileum
Postoperatively, child was stable with no further vomiting episodes, abdominal distention reduced and she started passing stool. She was started on feeds on post-operative day 2 , had no further vomiting episodes and was discharged on the third postoperative day. After probing the mother for history of foreign body, she revealed that child would be in her neighbor's house most of the time and another child there had fed small rubber balls to her 2 days before the onset of symptoms.

\section{DISCUSSION}

One of the major challenges in diagnosing foreign bodies in children is the lack of history in children as most of the foreign body ingestions occur to be accidental in nature [5] as in this case the child presented with absolutely no initial history of foreign body ingestion. Accidental foreign body ingestions are usually asymptomatic in about $20 \%$ of the cases [5]. The common symptoms include choking, refusal to eat, vomiting, drooling of saliva, wheezing, blood stained saliva, or respiratory distress. It was also been emphasized that one should consider the possibilities of peritonitis or small bowel obstruction due to a foreign body [6]. This is very much evidenced in the case presented here.

While X-ray can pick up most foreign bodies, a major diagnostic challenge is that foreign bodies made of plastic or wood are difficult to diagnose preoperatively unless they are coated with lead paint [6]. Oral contrast studies have the underlying risk of aspiration. Hence, this emphasizes the need for evaluation with other modalities such as ultrasound and computed tomography (CT) scan abdomen. Even though ultrasound examination may provide reasonable information about the sizes, structure, location of foreign bodies, and their relationship with other organs, a more accurate tool for the diagnosis of foreign body causing gastro-intestinal (GI) obstruction is a high-resolution computed tomography (HRCT) scan of abdomen [7]. In our case, we had to use X-ray, ultrasound as well as CT scan to confirm the small bowel obstruction caused by the mass and hence, the timely need for surgical intervention.

While around $90 \%$ of the GI foreign bodies pass through uneventfully, some foreign bodies that are too large to cross the pylorus and duodenal sweep need removal endoscopically in $10-20 \%$ of the cases and surgical intervention is needed in around $1 \%$ of the cases [8], as was the need in this case. While many reports have shown button batteries, magnets, sharp objects, coins, long objects, and absorptive objects as common foreign objects in gastric cavity and small bowel, there is a paucity of literature where rubber balls have been identified as a cause of small bowel obstruction resulting in illness and morbidity in the child requiring medical and surgical intervention.

\section{CONCLUSION}

This case highlights the need for a meticulous history taking, with the suspicion of a foreign body, especially when a toddler comes with persistent vomiting and also continuous monitoring, which would facilitate early intervention, thus reducing morbidity and 
mortality. Furthermore, an alert supervision of the toddlers by their caretakers is of utmost importance. It is also the pediatrician's responsibility to counsel the parents about constant supervision of their children, especially toddlers.

\section{REFERENCES}

1. Komninos ID, Tsiligianni IG. Foreign body ingestion mimicking irritable bowel syndrome: A case report. J Med Case Rep 2010;4:244.

2. Alsharief AN, Blackmore C, Schmit P. Small bowel obstruction due to ingestion of rubber balls. Pediatr Radiol 2017;47:1539-41.

3. Anderson JE, Brown EG, Greenholz SK. Multifocal small bowel obstruction in an infant. J Pediatr Surg 2015;50:1413-4.

4. Bradford V, Vadi M, Carter H. Diagnosis and management of a postpyloric foreign body causing small bowel obstruction in an infant. Clin Med Insights 2017;10:1-4.

5. Bakhshaeekia A, Hosseini SM, Razmi T, Shamsaeefar A. Bowel perforation by crumpled paper in a patient presenting with acute abdominal pain. Saudi J Gastroenterol 2009;15:52-4.

6. Ikenberry SO, Jue TL, Anderson MA, Appalaneni V, Banerjee S, BenMenachem T, et al. ASGE standards of practice committee management of ingested foreign bodies and food impactions. Gastrointest Endosc 2011;73:1085-91.

7. Miniter BM, Arruda AG, Zuckerman J, Caceres AV, Ben-Amotz R. Use of computed tomography (CT) for the diagnosis of mechanical gastrointestinal obstruction in canines and felines. PLoS One 2019;14:e219748.

8. Sai Prasad TR, Low Y, Tan CE, Jacobsen AS. Swallowed foreign bodies in children: Report of four unusual cases. Ann Acad Med Singap 2006;35:49-53.

Funding: None; Conflicts of Interest: None Stated.

How to cite this article: Rashmi N, Yerrapragada DB, Kumar MGA. Vomiting in a toddler - An unsuspected cause. Indian J Child Health. 2021; 8(10):371-373. 